\title{
Transfer learning for informative-frame selection in laryngoscopic videos through learned features
}

\author{
Ilaria Patrini* . Michela Ruperti* . \\ Sara Moccia - Leonardo S. Mattos . \\ Emanuele Frontoni • Elena De Momi
}

Received: date / Accepted: date

\begin{abstract}
Narrow-band imaging (NBI) laryngoscopy is an optical-biopsy technique used for screening and diagnosing cancer of the laryngeal tract, reducing the biopsy risks but at the cost of some drawbacks, such as large amount of data to review to make the diagnosis. The purpose of this paper is to develop a deep-learning-based strategy for the automatic selection of informative laryngoscopic-video frames, reducing the amount of data to process for diagnosis.
\end{abstract}

I. Patrini, M. Ruperti, E. De Momi

Department of Electronics, Information and Bioengineering, Politecnico di Milano piazza Leonardo Da Vinci 32

Milan (Italy)

S. Moccia

Department of Information Engineering, Università Politecnica delle Marche via Brecce Bianche 12

Ancona (Italy)

Department of Advanced Robotics, Istituto Italiano di Tecnologia

via Morego 30

Genoa (Italy)

L. S. Mattos

Department of Advanced Robotics, Istituto Italiano di Tecnologia

via Morego 30

Genoa (Italy)

E. Frontoni

Department of Information Engineering, Università Politecnica delle Marche via Brecce Bianche 12

Ancona (Italy)

* These authors equally contributed to this paper 
The strategy leans on the transfer learning process that is implemented to perform learned-features extraction using six different convolutional neural networks $(\mathrm{CNNs})$ pre-trained on natural images. To test the proposed strategy, the learned features were extracted from the NBI-InfFrames dataset. Support vector machines (SVMs) and CNN-based approach were then used to classify frames as informative (I) and uninformative ones such as blurred (B), with saliva or specular reflections $(\mathrm{S})$ and underexposed (U).

The best-performing learned-feature set was achieved with VGG 16 resulting in a recall of I of 0.97 when classifying frames with SVMs and 0.98 with the CNN-based classification. This work presents a valuable novel approach towards the selection of informative frames in laryngoscopic videos and a demonstration of the potential of transfer learning in medical image analysis.

Keywords Informative-frame selection · learned features · deep learning · transfer learning · laryngoscopy

\section{Introduction}

Optical imaging, such as microscopy and endoscopy, supports clinicians and surgeons in performing diagnosis and treatment [1]. Tissue analysis from optical images is crucial in several fields, such as ophthalmology [2, 3], laryngology $[4,5,6]$, and neurosurgery $[7,8]$. The automatic image-based classification of tissues is a valuable solution to offer surgical decision support.

The surgical data science (SDS) community is focusing more and more onto approaches to perform tissue classification in the operating room (OR) explointing machine learning (ML). In this paper we address the issue of enabling the application of SDS methods in laryngology where, however, different aspects can hamper a robust tissue classification, such as the presence of noise in the image, different camera pose with respect to the tissues, varying illumination level, and intra- and inter-patient tissue variability. In literature, there are several work that try to overcome these issues. For example, [5] tackles the challenge of intra- and inter-patient tissue variability providing a valuable method to perform reliable tissue classification in laryngoscopy images exploiting texture information and support vector machines (SVMs). In [48], the authors faced the issue of noise in the images proposing the creation of panorama of the larynx for inspection, which was obtained through an automatic frame selection strategy that discarded non-informative frames. In [41] the objective of the work was to automatically characterize and assess vascular patterns in contact endoscopy combined with narrow band imaging images to classify images based on the vascular pattern and laryngeal histopathology.

The output of SDS tools, in the field of laryngology, can be strongly affected by the quality of the laryngoscopic video frames. Indeed, the analysis of lowquality uninformative frames during endoscopy-based diagnosis, may increase the overall computational time required by SDS algorithms without providing any useful information. Moreover, there could be wrong classification outcomes 
Table 1 State-of-the-art approaches to informative-frame selection.

\begin{tabular}{|c|c|c|c|c|}
\hline Method & Year & Anatomical district & Feature set & Classification \\
\hline Bashar et al. [19] & 2010 & Gastro-intestinal tract & Intensity and texture & $\begin{array}{l}\text { Support vector } \\
\text { machines }\end{array}$ \\
\hline Atasoy et al. [20] & 2012 & Gastro-intestinal tract & $\begin{array}{l}\text { Image power-spectrum } \\
\text { histogram }\end{array}$ & Clustering \\
\hline Park et al. [21] & 2012 & Colon & Anatomy-related & $\begin{array}{l}\text { Conditional } \\
\text { random fields }\end{array}$ \\
\hline Maghsoudi et al. [22] & 2014 & Gastro-intestinal tract & Intensity & k-means \\
\hline Ishijima et al. [23] & 2015 & Oral cavity-esophagus & $\begin{array}{l}\text { Intensity, entropy } \\
\text { and keypoints }\end{array}$ & $\begin{array}{l}\text { Statistical } \\
\text { comparison }\end{array}$ \\
\hline Armin et al. [24] & 2015 & Colon & $\begin{array}{l}\text { Motion, intensity and } \\
\text { image derivatives }\end{array}$ & Random forest \\
\hline Perperidis et al. [12] & 2017 & Lungs & Texture & $\begin{array}{l}\text { Gaussian mixture } \\
\text { model }\end{array}$ \\
\hline Moccia et al. [11] & 2018 & Larynx & $\begin{array}{l}\text { Intensity, entropy, } \\
\text { keypoints and texture }\end{array}$ & $\begin{array}{l}\text { Support vector } \\
\text { machines }\end{array}$ \\
\hline
\end{tabular}

when processing frames with low informative content, such as image with insufficient illumination [9].

A possible solution to identify and discard uninformative images consists in performing preliminary visual assessment of image quality. However, this operation is qualitative, prone to human error and usually time consuming [10]. A reasonable alternative to visual assessment is the automatic selection of informative frames, which is however not always trivial due to variability in image characteristics (e.g., noise level and resolution), image acquisition protocols, and tissue anatomy $[11,12]$.

Thus, the specific aim of this work is to investigate if features learned with convolutional neural networks (CNNs), pre-trained on natural images, can be exploited for informative-frame selection in endoscopic videos. In particular, for feature classification, both support vector machines (SVMs) and CNNbased approaches are investigated.

The experimental analysis is performed on the NBI-InfFrames dataset, which has been recently proposed in [11] for NBI endoscopic video frames analysis and is available online ${ }^{1}$. Indeed, to the best of the authors' knowledge, it is the only labeled dataset that is publicly available in the field. All codes and CNN weights will be made publicly available upon publication of this work.

This paper is organized as follows: Sec. 2 surveys the approaches for informativeframe selection, Sec. 3 explains the proposed approaches to informative-frame selection. Sec. 4 deals with the experimental protocol used to test the proposed methodology. Results are presented in Sec. 5 and discussed in Sec. 6. Finally, Sec. 7 summarizes the main achievements of this work.

1 DOI: $10.5281 /$ zenodo. 1162784 


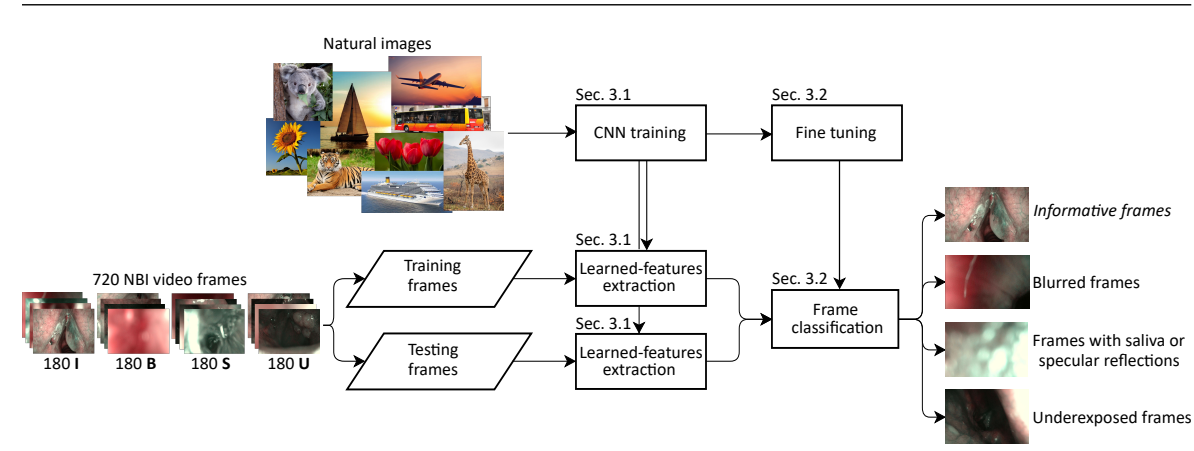

Fig. 1 Workflow of the proposed approach to informative frame selection in endoscopic videos in narrow-band imaging (NBI). Frames are classified in I: informative, B: blurred, $\mathbf{S}$ : with saliva and specular reflections, $\mathbf{U}$ : underexposed. The approach leans on transfer learning, which is implemented to perform learned-features extraction using different convolutional neural networks (CNNs) pre-trained on natural images. Frame classification is then performed following two different approaches, i.e. exploiting two different classifiers: support vector machines (SVMs) and fine-tuned CNN.

\section{Related work}

To accomplish the task of identify and discard uninformative images in endoscopic videos, several machine-learning (ML) approaches have been proposed, which are mainly based on handcrafted features (e.g., features based on intensity or textural information). Anatomical features are used in [21] to classify informative frames in colonoscopy videos with conditional random fields, while motion, edge and color features, along with random forests, are used in [24]. Frequency features, (i.e. power spectrum) from gastro-intestinal images are clustered with k-means in [20, 22], while in [19] local color histogram features are classified with SVMs. A statistical approach to informative-frame selection in esophageal microscopy images, which exploits intensity, entropy and keypoint-based features, is proposed in [23]. Texture-based features from lung microscopy images are classified with Gaussian mixture models in [12]. In [11], a set of intensity, keypoint-based and textural features and multi-class SVMs are used to classify informative and three classes of uninformative frames in laryngoscopic videos in narrow-band imaging (NBI). Strategies proposed in literature for informative-frame selection include also simple uniform or random frame sampling. Samples include [29], where cytoscopy frames showing the internal urinary bladder wall are subsampled by a factor of four. This kind of informative-frame sampling is fast in terms of computational time but does not guarantee that all informative frames are extracted while removing the non-informative ones. Furthermore, a large number of redundant keyframes could be selected in long video segments with identical content. Table 1 summarizes state-of-the-art approaches to informative-frame selection.

It has been shown that deep-learning algorithms may outperform standard learning approaches for image analysis, as shown by researchers in other SDS fields $[13,14,15]$. With deep learning, handcrafted features are replaced by 
learned features, which are automatically learned during a training process (i.e. without the need of manually defining the mathematical formulation of the feature set) [16]. The most popular approach to automatic feature learning is using convolutional neural networks (CNNs), which showed remarkable performance in classifying skin cancers [14] and predicting cardiovascular risk factors from retinal fundus photographs [17]. A CNN is a neural network that consists of a series of different kinds of specialized layers, such as convolutional and pooling. The first (upper) CNN layers learn how to extract image features directly from the training images, thus the training set is commonly made of million of images to satisfactorily encode variability in the images (e.g., ImageNet [18], a dataset for natural-image classification, is made of more of 14-million images).

Collecting such a high number of labeled images is challenging in the medical field [1], despite the efforts of international organizations ${ }^{2}$.

This problem may be overcome through transfer learning, whose fundamental motivation in machine learning field was discussed in a NIPS-95 workshop on "Learning to Learn". Unlike traditional ML techniques that try to learn each task from scratch, transfer learning enables the intelligent use of knowledge and skills learned in a different scenario to solve novel tasks with good performance [31]. Thus, on this basis, the aim of transfer learning is to apply the knowledge stored by the CNNs while solving one problem (e.g., natural image classification) to a different one (such as medical image classification) $[17,14]$. Transfer learning research has attracted growing interest since 1995. It has been applied in a variety of application areas (e.g., identification of tiny faces in thermal images [45], WiFi localization [47], reinforcement learning [46]). Moreover, it has already been shown to be successful also in several medical fields $[25,16,26]$ such as the classification of chronic obstructive pulmonary disease [27] or colorectal polyps [28], the prediction of skeletal muscle forces [44], mild depression recognition [43]. However, no application can be found in the field of informative-frame selection.

\section{Methods}

In this section, the proposed strategies to feature extraction (Sec. 3.1) and classification (Sec. 3.2) are explained. The workflow of the proposed approach is shown in Fig. 1.

\subsection{Transfer learning for learned-features extraction}

In this paper, learned-feature extraction was performed exploiting a transferlearning approach that focuses on storing the knowledge or weights of a trained neural network so that it can be reused for a further task [30].

2 https://grandchallenges.org/ 
Table 2 Tested convolutional neural networks (CNNs) and the corresponding number of learned features. Top- 1 and top- 5 accuracies achieved on the ImageNet dataset are reported too. These accuracies refer to the fractions of test images for which the correct label is the first (top-1) or among the five labels (top-5) considered most probable by the model, respectively.

\begin{tabular}{cccc}
\hline CNNs & Number of features & Top-1 accuracy & Top-5 accuracy \\
\hline VGG 16 & 4096 & $71.5 \%$ & $89.8 \%$ \\
Inception V4 & 1536 & $80.2 \%$ & $95.2 \%$ \\
ResNet V1 101 & 2048 & $76.4 \%$ & $93.2 \%$ \\
ResNet V1 152 & 2048 & $76.8 \%$ & $92.9 \%$ \\
ResNet V2 152 & 2048 & $87.8 \%$ & $94.1 \%$ \\
Inception - ResNet V2 & 1536 & $80.4 \%$ & $95.3 \%$ \\
\hline
\end{tabular}

Practically, it generalizes the knowledge (features, weights) of an existing solution to a new problem, leading to promising results also when the new task has significantly less data. This is a powerful method in the computer vision domain that enables the use of knowledge acquired for one task to solve related ones. This relies upon the fact that functionalities regarding the extraction of low-level features (e.g., edges, shapes, and corners) can be shared across tasks.

As reported in [31], the formal definition of transfer learning involves the concepts of a domain and a task. A domain $\mathcal{D}$ consists of a feature space $\mathcal{X}$ and a marginal probability distribution $P(X)$ over the feature space, where $\mathrm{X}$ $=\left\{x_{1}, \ldots, x_{n}\right\} \in \mathcal{X}$. Given a specific domain, $\mathcal{D}=\{\mathcal{X}, P(X)\}$, a task consists of two components: a label space $\mathcal{Y}$ and an objective predictive function $f(\cdot)$ (denoted by $\mathcal{T}=\{\mathcal{Y}, f(\cdot)\}$ ), which is not observed but can be learned from the training data, which consist of pairs $\left\{x_{i}, y_{i}\right\}$, where $x_{i} \in \mathrm{X}$ and $y_{i} \in \mathcal{Y}$.

Given a source domain $\mathcal{D}_{\mathcal{S}}$ and learning task $\mathcal{T}_{\mathcal{S}}$, a target domain $\mathcal{D}_{\mathcal{T}}$ and learning task $\mathcal{T}_{\mathcal{T}}$, transfer learning aims to help improve the learning of the target predictive function $f_{T}(\cdot)$ in $\mathcal{D}_{\mathcal{T}}$ using the knowledge in $\mathcal{D}_{\mathcal{S}}$ and $\mathcal{T}_{\mathcal{S}}$, where $\mathcal{D}_{\mathcal{S}} \neq \mathcal{D}_{\mathcal{T}}$, or $\mathcal{T}_{\mathcal{S}} \neq \mathcal{T}_{\mathcal{T}}$

Six pre-trained CNNs (Table 2) were investigated to perform learnedfeature extraction. The CNNs used were chosen among the best performing in the context of the Large Scale Visual Recognition Challenge (ILSVRC) ${ }^{3}$. For fair comparison, all the CNN models were pre-trained on the ImageNet dataset $^{4}$.

The tested CNN architectures, hereafter briefly described to highlight their main peculiarities, were:

$\boldsymbol{V G G} 16$ VGG 16 was proposed by the Oxford's Visual Geometry Group (VGG) in the context of ILSVRC in 2014.

VGG 16 improved the performance of previously proposed deep networks (e.g., AlexNet [32]) by replacing large-sized kernel filters with stacked kernels with dimension $3 \times 3$ pixels. The multiple stacked small-sized kernels allowed

\footnotetext{
3 http://www.image-net.org/challenges/LSVRC/

4 http://www.image-net.org/
} 
increased performance by enabling the VGG 16 to learn complex and fine-level features while making the training convergence easier and faster [33].

VGG 16 has a uniform (serial) architecture with 13 convolutional and 5 (down-sampling) max-pooling layers, followed by 3 fully-connected layers. The number of layer channels starts from 64 filters and increases by a factor of 2 after every pooling layer.

VGG 16 achieved the top-1 accuracy of $71.5 \%$ and the top-5 accuracy of $89.8 \%$ on the ImageNet dataset, where the top- 1 and top- 5 accuracies are the fractions of test images for which the correct label is the first (top-1) or among the five labels (top-5) considered most probable by the model, respectively.

Inception V4 The winner of ILSVRC 2014 competition was GoogLeNet (i.e., Inception V1) developed by Google LLC.

The innovative idea of GoogLeNet is the introduction of the Inception module. The input image to the module is convolved with parallel filters of different sizes $(1 \times 1,3 \times 3,5 \times 5)$, thus losing the CNN linear structure (such the one of VGG 16), to allow a multi-scale feature extraction. Several versions of the Inception module were proposed and the upgraded version Inception V4 was used here, as it showed the best performance [34].

The Inception V4 architecture is organized in 10 blocks, for a total of 14 inception modules linearly stacked with global average pooling at the end. The 14 Inception modules are different in terms of convolutional-kernel size, number of filters and depth. This allows to process the image at varying scale when it passes through the CNN modules.

Top- 1 and top-5 accuracies of Inception V4 of $80.2 \%$ and $95.2 \%$ were achieved on the ImageNet dataset, respectively.

ResNet V1 101 and ResNet V1 152 The Residual Neural Networks (ResNets) presented in [35] got the first place in the ILSVRC 2015 classification competition and the first place in ILSVRC and COCO 2015 competition in ImageNet Detection, ImageNet localization, Coco detection and Coco segmentation [35].

ResNets were introduced to solve the vanishing gradient and the gradation problem that arise when training ultra-deep CNNs. They consist of many stacked residual units (building blocks) containing skip connections to link the input and output of each unit. CNNs with residual units were shown to outperform their plain counterparts [35].

In this work, ResNets with 101 layers and 152 layers were tested. ResNet 101 consists of 4 main layers and the number of building blocks varies in each layer $(3,4,23$ and 3, respectively). Each building block is made of 3 convolutional kernels with the skip connection. ResNet 152 has the same structure but with 8 and 36 building blocks in the second and third layer, respectively. Similarly to Inception V4, both ResNets end with a global average pooling followed by a classification layer. 
Table 3 Tested conditions in this work. Condition 1 (C1) exploited the convolutional neural networks $(\mathrm{CNNs})$ as features extractor and the extracted learned features were then classified by means of support vector machines (SVMs); in condition 2 (C2) the best performing CNN (from C1 experiments) was fine-tuned and it is used both as feature extractor and classifier.

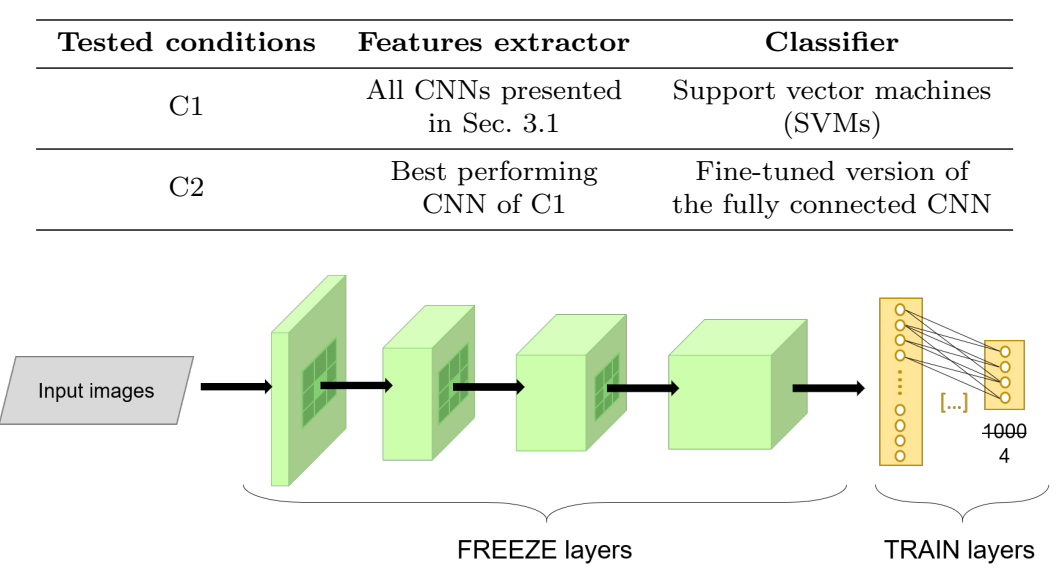

Fig. 2 Graphical representation of the fine-tuning technique. Weights of the first layers are frozen, since they refer to general features, while the weights of the last layers (or at least the ones of the fully-connected layer) are learnt on the target dataset.

ResNet V1 101 and ResNet V1 152 achieved top-1 accuracies of $76.4 \%$ and $76.8 \%$ and top- 5 accuracies of $93.2 \%$ and $92.9 \%$, respectively on the ImageNet dataset.

ResNet V2 152 ResNet V2 was introduced in [36] with the goal of using pre-activation in ResNets. Pre-activation consists in using activation functions (such as the ReLU) as pre-activation of the convolutional layers, in contrast to conventional ResNets where the activation functions are used as postactivation. Pre-activation was demonstrated to have an impact both in terms of ease of optimization and improved regularization.

The version of ResNet V2 with 152 layers was tested here, which is ResNet V1 152 with pre-activation. It achieved top- 1 and top- 5 accuracy of $87.8 \%$ and $94.1 \%$ on ImageNet, respectively.

Inception - ResNet V2 A hybrid Inception module was proposed in [34] by Szegedy et al., which is called Inception - ResNet V2.

This architecture significantly improved the recognition performance of both ResNet V2 and Inception V4, and dramatically increased the training speed when tested on ImageNet dataset [34]. Inception - ResNet V2 was built by adding residual connections to link the input and output of the Inception V4 blocks.

Top-1 and top-5 validation accuracies of $80.4 \%$ and $95.3 \%$ were achieved on ImageNet, respectively. 


\subsection{Frame classification}

Learned-feature matrices were standardized before classification [37]. Feature classification was first performed exploiting SVMs [37] (C1 in Table 3) to tackle the high-dimensionality of the input features $(>1500)$ while being robust to noise in the features [38]. SVMs with Gaussian kernel $(\Psi)$ were used to prevent parameter proliferation, limiting the computational complexity. To implement multi-class SVM classification, the one-vs-rest scheme was used. Thus, when one class was considered positive, the remaining ones were considered negative.

The SVM hyperparameters, i.e. kernel coefficient $(\gamma)$ and penality parameter $(C)$, were retrieved via grid-search and cross-validation as explained in Sec. 4.

We also investigated the performance of the CNNs using the best-performing learned-feature set for frame classification (C2 in Table 3). To this goal, finetuning was implemented by freezing the weights of the first CNN layers and learning the layers of the fully-connected layers [39], as reported in Fig. 2. Indeed, the first layers contain more generic features (e.g. edge detectors or color blob detectors) that should be useful to many tasks, while the last layers become progressively more specific to the details of the classes contained in the original dataset [13]. In order to accomplish this task, Gradient Descent Optimizer (GDO) was used. The exploited optimizer relied upon the stochastic gradient descent (SGD) algorithm and it has been chosen since, as observed in [49], solutions found by standard SGD generalize better than adaptive methods (e.g., Adam Optimizer). During training, the categorical cross-entropy was minimized. It is defined as:

$$
C E=-\sum_{i=i}^{C} t_{i} \log \left(s_{i}\right)
$$

being $t_{i}$ and $s_{i}$ the ground truth and the CNN score for each class $i$ in $C \in$ $[1,4]$, respectively.

\subsection{Frame classification with training from scratch}

To prove and quantify the transfer learning effect, we performed an additional experiment where the best performing architecture was trained from scratch. Weights initialization was performed with Glorot initialization [42], i.e. weights are initialized as random draws from a truncated normal distribution with 0 mean and standard deviation

$$
\sigma=\sqrt{\frac{2}{\text { fan }_{\text {in }}+\text { fan }_{\text {out }}}}
$$

being $f_{a n}$ and fan $_{\text {out }}$ the number of input and output units, respectively. 


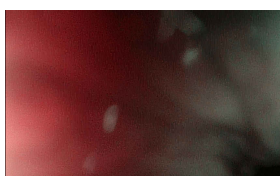

(a) $\mathbf{B}$

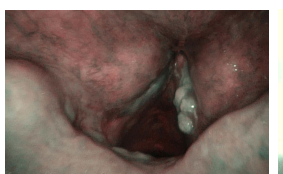

(b) $\mathbf{I}$

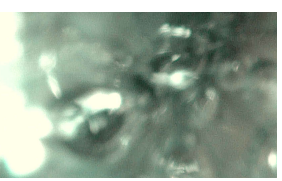

(c) $\mathbf{S}$

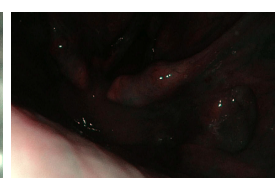

(d) $\mathbf{U}$

Fig. 3 Samples of laryngeal video frames in the NBI-InfFrames. Frames were B: blurred, I: informative, S: with saliva and specular reflections, U: underexposed.

\section{Experimental protocol}

\subsection{Dataset}

The performance of the learned features extracted with the CNNs presented in Sec. 3.1 were evaluated on the NBI-InfFrames (as introduced in Sec. 1), which was built from 18 NBI endoscopic videos, referring to 18 different patients affected by squamous cell carcinoma (SCC). All videos were acquired with a NBI endoscopic system (Olympus Visera Elite S190 video processor and an ENF-VH rhino-laryngo videoscope) with frame rate of $25 \mathrm{fps}$ and image size of $1920 \times 1072$ pixels.

The NBI-InfFrames consists of a total of 720 video frames, which are equally divided in four classes: informative $(\mathbf{I})$, blurred $(\mathbf{B})$, with saliva or specular reflections $(\mathbf{S})$, and underexposed $(\mathbf{U})$. For each of the 18 NBI endoscopic videos, video frames were randomly extracted and presented to two human evaluators first. Then, the two evaluators were asked to label the frames. In case the two evaluators did not agree on the class, a third evaluator was asked to choose the ultimate class among the two proposed by the two evaluators. This process was repeated until all the 720 frames were extracted from the videos. For the manual labeling process, the following set of rules was defined:

- I frames should have an adequate exposure and clearly visible blood vessels; they may also present micro-blur and small portions of specular reflections (up to $10 \%$ of the image area).

- B frames should show a homogeneous and widespread blur.

- $\mathbf{S}$ frames should present bright white/light-green bubbles or blobs, overlapping with at least half of the image area.

- U frames should present a high percentage of dark pixels, even though small image portions (up to $10 \%$ of the image area) with over- or normal exposure are allowed.

All the videos composing the NBI-InfFrames dataset are rearranged in three subfolders used for cross-validation purposes during the frame classification performance assessment. As reported in Table 4.1, data separation in the folds is performed both at class- and frame-level because each fold contains the same number of video frames for each of the four classes (i.e. 60) and the frames belonging to a patient contribute to build one fold only. It was not always possible, for some videos, to select an equal number of frames for the four 
Table 4 NBI-InfFrames evaluation dataset. It is reported the number of frames that contributed to build the dataset, for each video (video ID) and for each class (I, B, S, U). The dataset is split in 3 folds to perform robust estimation of the classification performance. I: informative frame; B: blurred frame; $\mathbf{S}$ : frame with saliva or specular reflections; $\mathbf{U}$ : underexposed frame.

\begin{tabular}{|c|c|c|c|c|c|}
\hline & Video ID & I & B & $\mathbf{S}$ & $\mathbf{U}$ \\
\hline \multirow[t]{6}{*}{ Fold 1} & 1 & 10 & 10 & 20 & 11 \\
\hline & 2 & 10 & 0 & 6 & 9 \\
\hline & 3 & 10 & 0 & 0 & 2 \\
\hline & 4 & 10 & 40 & 23 & 20 \\
\hline & 5 & 10 & 10 & 11 & 3 \\
\hline & 6 & 10 & 0 & 0 & 15 \\
\hline$\sum$ & 6 & 60 & 60 & 60 & 60 \\
\hline \multirow[t]{6}{*}{ Fold 2} & 7 & 10 & 28 & 19 & 0 \\
\hline & 8 & 10 & 8 & 21 & 5 \\
\hline & 9 & 10 & 3 & 10 & 10 \\
\hline & 10 & 10 & 21 & 10 & 16 \\
\hline & 11 & 10 & 0 & 0 & 14 \\
\hline & 12 & 10 & 0 & 0 & 15 \\
\hline$\sum$ & 6 & 60 & 60 & 60 & 60 \\
\hline \multirow[t]{6}{*}{ Fold 3} & 13 & 10 & 17 & 0 & 10 \\
\hline & 14 & 10 & 21 & 34 & 22 \\
\hline & 15 & 10 & 0 & 11 & 10 \\
\hline & 16 & 10 & 0 & 9 & 5 \\
\hline & 17 & 10 & 12 & 0 & 2 \\
\hline & 18 & 10 & 10 & 6 & 11 \\
\hline$\sum$ & 6 & 60 & 60 & 60 & 60 \\
\hline
\end{tabular}

classes, particularly for non-informative ones. This because either a sufficient number of frames was not available for all the classes or the videos could contribute only with frames with mixed characteristics among the four classes. When this was the case, the other videos in the fold contributed to balance the number of frames. Sample images for the four classes are shown in Fig. 3.

In addition to this, one of the $18 \mathrm{NBI}$ endoscopic videos was manually annotated. The video was $18.24 \mathrm{~s}$ long with $29 \mathbf{B}$ frames, $302 \mathbf{I}$ frames, $100 \mathbf{S}$ frames and $25 \mathbf{U}$ frames.

\subsection{Parameters Tuning}

\subsubsection{Feature extraction}

To extract learned features, from each video frame, with the architectures presented in Sec. 3.1, all the images were resized to match the input size of the investigated CNN architectures. The deepest layer of each tested CNN was considered as feature extractor. The feature length for each feature set is shown in Table 2. 


\subsubsection{Classification}

For performing the classification with SVM, $\gamma$ and $C$ (the SVM hyper-parameters) were retrieved via grid-search and 10-fold cross validation. The grid-search spaces for $\gamma$ and $C$ were set to $\left[10^{-8}, 10\right]$ and $\left[10^{-3}, 10^{6}\right]$, respectively, with 10 values evenly spaced on $\log _{10}$ scale in both cases.

For performing the CNN-based classification, fine tuning was implemented using the GDO with a learning rate of $10^{-5}$.

To estimate performances, 3 -fold cross-validation was performed separating data at patient level, as suggested in [11]. When the classification of the frames in fold 1 (i.e. 240 images) was performed, folds 2 and 3 (i.e. 480 images) were used for training. The same has been done for testing the classification of frames in fold 2 and 3, using fold 1 and 3 , and fold 1 and 2 for hyper-parameter tuning, respectively. To be consistent with the aforementioned approach, the cross-validation of the fine-tuning technique has been done in the same fashion.

The classification of the fully labeled video sequence was performed with the fine-tuned CNN, where all the frames belonging to the three folds (excluding the ones of the fully labeled video) were used for training, for a total of 663 training frames.

For fair comparison, 3-fold cross validation was used for testing purposes also when testing the performance of the CNN trained from scratch.

\subsection{Data analysis}

The classification performance of each CNN model was evaluated computing the class-specific recall $\left(\boldsymbol{R e c}_{\text {class }}=\left\{\operatorname{Rec}_{\text {class }_{j}}\right\}_{j \in[1,4]}\right)$, the precision $\left(\mathbf{P r e c}_{\text {class }}\right.$ $\left.=\left\{\text { Prec }_{\text {class }_{j}}\right\}_{j \in[1,4]}\right)$, the F1 score $\left(\mathbf{F} \mathbf{1}_{\text {class }}=\left\{F 1_{\text {class }_{j}}\right\}_{j \in[1,4]}\right)$, where:

$$
\begin{gathered}
\operatorname{Rec}_{\text {class }_{j}}=\frac{T P_{j}}{T P_{j}+F N_{j}} \\
\operatorname{Prec}_{\text {class }_{j}}=\frac{T P_{j}}{T P_{j}+F P_{j}} \\
F 1_{\text {class }_{j}}=2 \frac{\operatorname{Prec}_{\text {class }_{j}} \times \operatorname{Rec}_{\text {class }_{j}}}{\operatorname{Prec}_{\text {class }_{j}}+\operatorname{Rec}_{\text {class }_{j}}}
\end{gathered}
$$

being $T P_{j}$ the number of true positive of the $j^{\text {th }}$ class, $F N_{j}$ the number of false negative of the $j^{t h}$ class and $F P_{j}$ the number of false positive of the $j^{t h}$ class.

The area (AUC) under the receiver operating characteristic (ROC) was used to evaluate classification performance because, unlike confusion-matrix based measures (e.g. accuracy), it describes the discriminatory power of a classifier independently of the class distribution [50]. It was computed from the probability threshold resulting from the CNNs. As the classification problem was a multi-class problem (with a balanced dataset), to compare the different $\mathrm{CNN}$ approaches to learned-feature extraction we computed the macro-average 
ROC, which is an extent of the ROC curve and computes the metric independently for each class and then it takes the average. For the best-performing feature set, i.e. the one that gave the highest recall for I, we performed the ROC analysis for each class.

The features learned with the investigated CNN architectures were compared with the set of features proposed in [11] in terms of classification performance. Only the method presented in [11] was considered, as it had already been shown to outperform previous literature on the topic. For the sake of completeness, we used the Wilcoxon signed-rank test (significance level $=0.05$ ) for paired sample to assess whether the classification achieved with our best performing feature vector significantly differs from the ones achieved with the other feature sets.

Feature extraction and feature classification were implemented with Tensorflow $^{5}$ and scikit-learn ${ }^{6}$, respectively. All the TensorFlow CNN-model files and the CNN weights were downloaded from the TensorFlow-Slim image classification model library ${ }^{7}$. The network's fine tuning was implemented with Keras $^{8}$ with TensorFlow backend.

Experiments were performed on Intel ${ }^{\circledR}$ Core TM i7-4500 CPU @ $1.80 \mathrm{GHz}$ - $2.40 \mathrm{GHz}$ with $8 \mathrm{~GB}$ of available RAM; NVIDIA GeForce GT $740 \mathrm{M}$; Microsoft Windows 10 64-bit operating system, availing of Google Colaboratory (a.k.a Colab), a cloud service based on a Jupyter notebook environment that supports $14858 \mathrm{MB}$ of free GPU, $12.4 \mathrm{~GB}$ of free RAM and a processor size of 900.6 MB.

\section{Results}

For SVM-based classification (C1 in Table 3), the macro-averaging ROC for the investigated CNN architectures are shown in Fig. 4. With the bestperforming learned-feature set (obtained with VGG 16), an AUC of 0.9856 was achieved.

The ROC curves relative to each of the four frame classes for the VGG 16-based feature set are shown in Fig. 5(a). AUC values were 0.9973 for informative frames $(\mathbf{I}), 0.9881$ for blurred frames $(\mathbf{B}), 0.9862$ for frames with saliva or specular reflections $(\mathbf{S})$ and 0.9852 for underexposed frames $(\mathbf{U})$.

From the confusion matrix relative to VGG 16 in Fig. 6(a), Rec of 0.9722 was achieved for $\mathbf{I}, 0.9611$ for $\mathbf{B}, 0.8778$ for $\mathbf{S}$ and 0.9333 for $\mathbf{U}$. The median Rec among the four classes was 0.9361 . Misclassification mainly occurred between $\mathbf{S}$ and $\mathbf{I}$, probably due to the presence of image-intensity edges in $\mathbf{S}$ frames (e.g., saliva blobs and specular reflections) as in $\mathbf{I}$ frames.

\footnotetext{
5 http://www.tensorflow.org

6 http://scikit-learn.org

7 https://github.com/tensorflow/models/tree/master/research/slim

8 https://keras.io
} 
Table 5 Support vector machines (SVMs)-based classification performance in terms of class-specific precision $\left(\mathbf{P r e c}_{\text {class }}\right)$, recall $\left(\mathbf{R e c}_{\text {class }}\right)$ and F1-score $\left(\mathbf{F} \mathbf{1}_{\text {class }}\right)$ are reported for the four different classes. B: blurred frames, $\mathbf{I}$ : informative frames, $\mathbf{S}$ : frames with saliva or specular reflections, U: underexposed frames. Results from the state of art [11] report only two significant digits.

\begin{tabular}{|c|c|c|c|}
\hline & Prec $_{\text {class }}$ & Rec $_{\text {class }}$ & F1 $1_{\text {class }}$ \\
\hline \multicolumn{4}{|c|}{ Moccia et al., 2018 [11] } \\
\hline $\mathbf{B}$ & 0.76 & 0.83 & 0.79 \\
\hline I & 0.91 & 0.91 & 0.91 \\
\hline $\mathbf{S}$ & 0.78 & 0.62 & 0.69 \\
\hline $\mathbf{U}$ & 0.76 & 0.85 & 0.80 \\
\hline avg/total & 0.80 & 0.80 & 0.80 \\
\hline \multicolumn{4}{|l|}{ Inception V4 } \\
\hline $\mathbf{B}$ & 0.8883 & 0.9722 & 0.9284 \\
\hline I & 0.9441 & 0.8444 & 0.8915 \\
\hline $\mathbf{S}$ & 0.9012 & 0.8611 & 0.8807 \\
\hline $\mathbf{U}$ & 0.8526 & 0.9000 & 0.8757 \\
\hline avg/total & 0.8966 & 0.8944 & 0.8941 \\
\hline \multicolumn{4}{|c|}{ Inception-ResNet V2 } \\
\hline $\mathbf{B}$ & 0.8737 & 0.9611 & 0.9153 \\
\hline I & 0.9571 & 0.8667 & 0.9096 \\
\hline $\mathbf{S}$ & 0.8824 & 0.8333 & 0.8571 \\
\hline $\mathbf{U}$ & 0.8836 & 0.9278 & 0.9051 \\
\hline avg/total & 0.8992 & 0.8972 & 0.8968 \\
\hline \multicolumn{4}{|c|}{ ResNet V1 101} \\
\hline B & 0.8947 & 0.9444 & 0.9189 \\
\hline $\mathbf{I}$ & 0.9824 & 0.9278 & 0.9543 \\
\hline $\mathbf{S}$ & 0.9128 & 0.8722 & 0.8920 \\
\hline $\mathbf{U}$ & 0.9415 & 0.9833 & 0.9620 \\
\hline avg/total & 0.9329 & 0.9319 & 0.9318 \\
\hline \multicolumn{4}{|c|}{ ResNet V1 152} \\
\hline B & 0.9259 & 0.9722 & 0.9485 \\
\hline $\mathbf{I}$ & 0.9881 & 0.9222 & 0.9540 \\
\hline $\mathbf{S}$ & 0.8913 & 0.9111 & 0.9011 \\
\hline $\mathbf{U}$ & 0.9441 & 0.9389 & 0.9415 \\
\hline avg/total & 0.9374 & 0.9361 & 0.9363 \\
\hline \multicolumn{4}{|l|}{ ResNet V2 152} \\
\hline $\mathbf{B}$ & 0.9198 & 0.9556 & 0.9373 \\
\hline I & 0.9603 & 0.8056 & 0.8761 \\
\hline $\mathbf{S}$ & 0.8316 & 0.9056 & 0.8670 \\
\hline $\mathbf{U}$ & 0.9140 & 0.9444 & 0.9290 \\
\hline avg/total & 0.9064 & 0.9028 & 0.9024 \\
\hline \multicolumn{4}{|l|}{ VGG 16} \\
\hline B & 0.9202 & 0.9611 & 0.9402 \\
\hline I & 0.9722 & 0.9722 & 0.9722 \\
\hline $\mathbf{S}$ & 0.9349 & 0.8778 & 0.9054 \\
\hline $\mathbf{U}$ & 0.9180 & 0.9333 & 0.9256 \\
\hline avg/total & 0.9363 & 0.9361 & 0.9359 \\
\hline
\end{tabular}




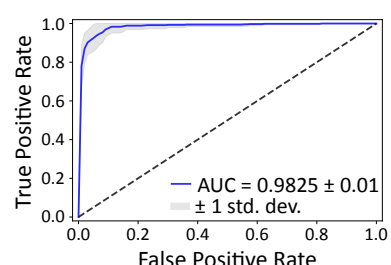

(a) Inception V4

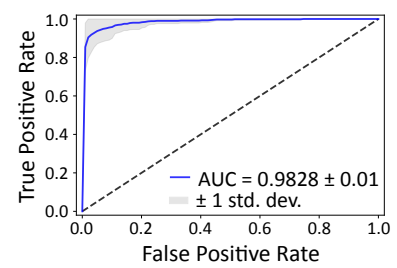

(d) ResNet V1 152

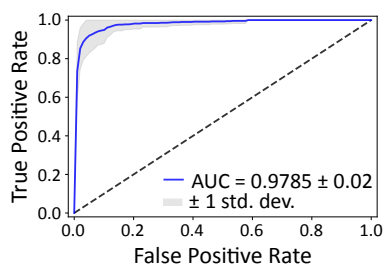

(b) Inception-ResNet V2

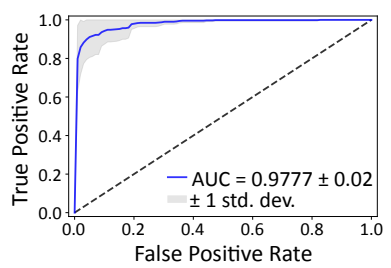

(e) ResNet V2 152

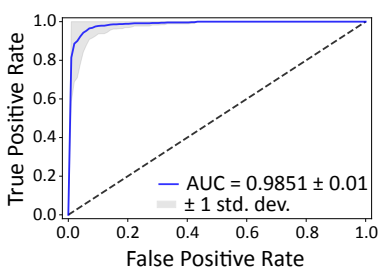

(c) ResNet V1 101

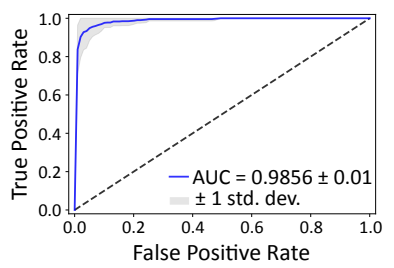

(f) VGG 16

Fig. 4 Macro-averaging receiver operating characteristic (ROC) curves for the investigated architectures coupled with support vector machines (SVMs). The area under the ROC (AUC) for each architecture is reported, too.

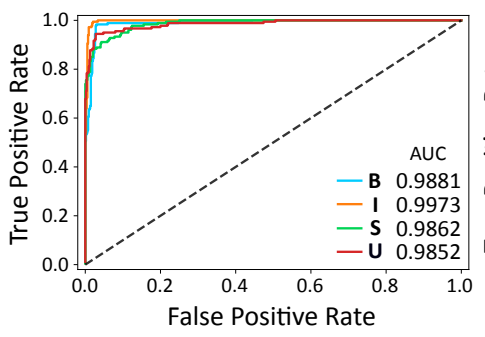

(a) VGG 16 (features) + SVM



(b) VGG 16

Fig. 5 Receiver operating characteristic (ROC) curves for the four frame classes obtained with VGG 16. Features learned with VGG 16 are classified with (a) support vector machines (SVM) and (b) fully-connected layers. The area under the ROC (AUC) for each class is reported, too. B: blurred frames, I: informative frames, $\mathbf{S}$ : frames with saliva or specular reflections, U: underexposed frames.

The fine-tuned VGG 16-based classification (C2 in Table 3) achieved values of 0.9778 for the Rec of I, 0.9333 for B, 0.8556 for $\mathbf{S}$ and 0.9389 for $\mathbf{U}$. The ROC curves relative to each of the four frame classes are shown in Fig. 5(b).

Fig. 7 reports the comparison in terms of $\mathbf{R e c}_{\text {class }}$ for the tested CNN architectures and the method presented in [11]. Learned features always outperformed the handcrafted ones proposed in [11]. AUC values obtained with VGG 16-based classification were 0.9928 for informative frames (I), 0.9736 for blurred frames (B), 0.9819 for frames with saliva or specular reflections $(\mathbf{S})$ and 0.9861 for underexposed frames $(\mathbf{U})$.

No significant differences were found when applying the Wilcoxon signedrank test ( $p$-value $>0.05$ ) to the $\mathbf{R e c}_{\text {class }}$ vector of the resulted best feature set 


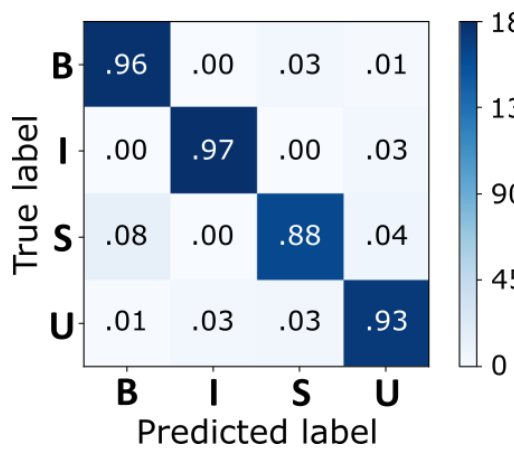

(a)

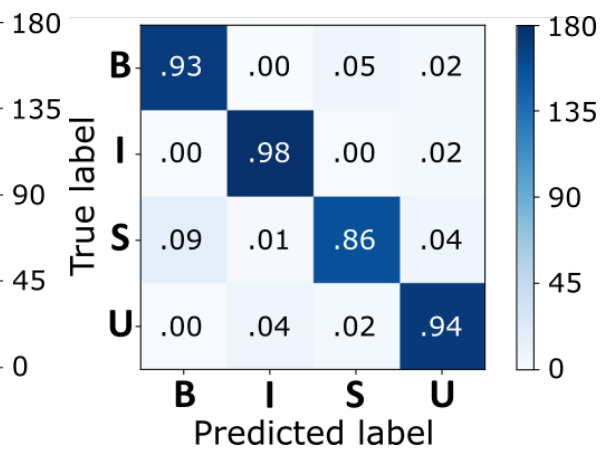

(b)

Fig. 6 Normalized confusion matrices for the best performing architecture (i.e., VGG 16): 6(a) SVMs and 6(b) fine-tuned CNN-based classification. B: blurred frames, I: informative frames, S: frames with saliva or specular reflections, $\mathbf{U}$ : underexposed frames. The colorbar indicates the number of images.

Table 6 Classification performance of VGG 16 trained from scratch. Performance are reported in terms of class-specific precision $\left(\mathbf{P r e c}_{\mathbf{c l a s s}}\right)$, recall $\left(\mathbf{R e c}_{\mathbf{c l a s s}}\right)$ and F1-score (F1 $\left.1_{\text {class }}\right)$ are reported for the four different classes. B: blurred frames, I: informative frames, $\mathbf{S}$ : frames with saliva or specular reflections, $\mathbf{U}$ : underexposed frames.

\begin{tabular}{cccc} 
& Prec $_{\text {class }}$ & Rec $_{\text {class }}$ & F1 $_{\text {class }}$ \\
\hline VGG 16 trained from scratch & & & \\
\hline B & 0.8432 & 0.8667 & 0.8548 \\
I & 0.8589 & 0.7778 & 0.8163 \\
S & 0.6903 & 0.7556 & 0.7215 \\
avg/total & 0.9371 & 0.9111 & 0.9239 \\
& & & \\
& 0.8324 & 0.8278 & 0.8291 \\
\hline
\end{tabular}

(i.e. VGG 16) and the $\mathbf{R e c}_{\text {class }}$ vector of all the other feature sets (including the state of the art). The same was observed when comparing the $\mathbf{R e c}_{\text {class }}$ vector of the resulted best feature set (i.e. VGG 16) and the $\mathbf{R e c}_{\text {class }}$ vector of its fine-tuned version.

The classification performance of VGG 16 trained from scratch are reported in Table 6 in terms of class-specific precision $\left(\mathbf{P r e c}_{\text {class }}\right)$, recall $\left(\mathbf{R e c}_{\text {class }}\right)$ and F1-score $\left(\mathbf{F} 1_{\text {class }}\right)$ for the four different classes. Fig. 8 shows the comparison between the results obtained with VGG 16 trained from scratch (Fig. 8(a)) and when implementing the transfer learning approach (Fig. 8(b)), where VGG 16 was pre-trained on the ImageNet dataset.

The complete video sequence classification achieved value of 0.9238 for the Rec of I. Misclassification mainly occurred between classes of uninformative frames: $25 \%$ of $\mathbf{S}$ and $7 \%$ of $\mathbf{B}$ were misclassified as $\mathbf{U}, 3 \%$ of $\mathbf{B}$ was misclassified as $\mathbf{S}$. 


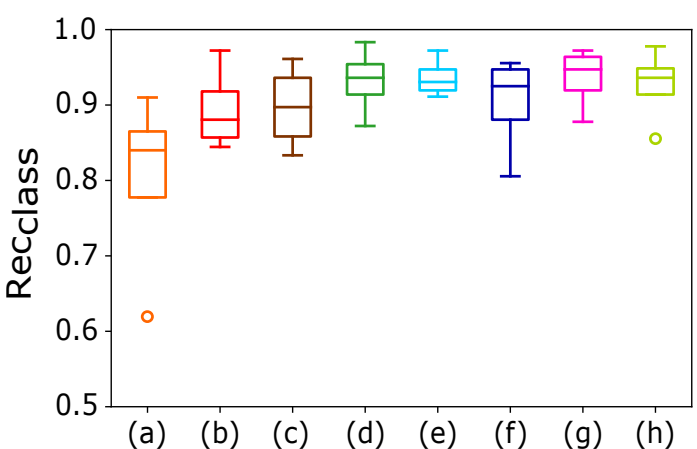

Fig. 7 Boxplots of classification recall (Rec class $_{\text {) }}$ ) obtained for (a) [11], for features classified with SVMs and extracted with (b) Inception V4, (c) Inception-ResNet V2, (d) ResNet V1 101, (e) ResNet V1 152, (f) ResNet V2 152, (g) VGG 16, and (h) for features extracted and classified with VGG 16 .

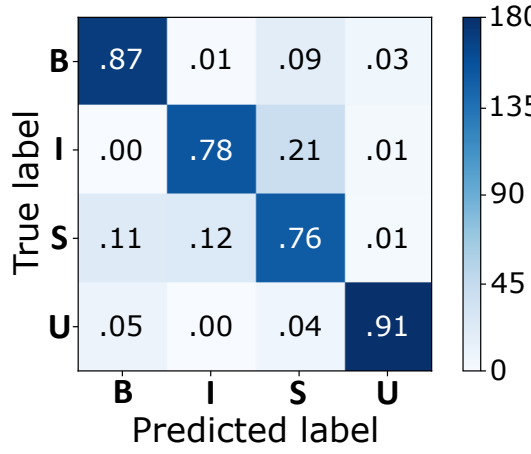

(a)

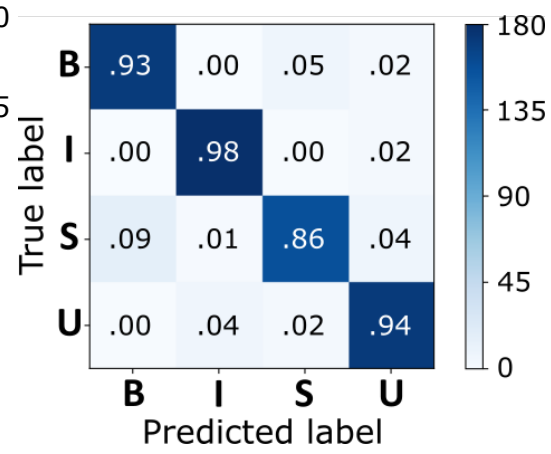

(b)

Fig. 8 Normalized confusion matrices for the VGG 16-based classification. 8(a): results obtained with a vanilla network (i.e. with a random initialization of the network's weights). 8(b): results obtained with a transfer learning approach, as described in Sec. 3.1. B: blurred frames, I: informative frames, $\mathbf{S}$ : frames with saliva or specular reflections, $\mathbf{U}$ : underexposed frames. The colorbar indicates the number of images.

\section{Discussion}

In this paper, we presented and evaluated a strategy for informative frame selection that exploits learned features automatically extracted from CNNs that were pre-trained on natural images.

When comparing the CNN architectures (C1 in Table 3), VGG 16 outperformed all the others in terms of AUC of the macro-average ROC curve and in terms of Rec of $\mathbf{I}$, as reported in Table 5. A visual comparison of the classifications of $\mathbf{I}$ by the investigated CNN models is reported in Table 7 . In Table 8, the number of misclassified $\mathbf{I}$ is reported, clearly showing that VGG 16 lowered the misclassification rate. The reason for this could be seen in the rel- 
atively simple (i.e., serial, without branches or skip connections) architecture and small depth (16 layers) of the VGG 16 architecture. This resulted in the extraction of more generalizable features and led to a more successful transfer learning.

From the comparison with the handcrafted-based approaches in the literature, and in particular with [11] (considered the state-of-the-art up to now as it outperformed all previously published methods), the learned features extracted with all the tested architectures showed higher $\mathbf{R e c}_{\text {class }}$ when classifying blurred frames, frames with saliva or specular reflections and underexposed frames. Moreover, three out of the six CNN architectures (i.e. ResNet V1 101, ResNet V1 152, and VGG 16) also showed higher value of Rec for informative frames. This confirmed considerations asserted in the literature of other SDS fields. In fact, deep-learning strategies coupled with transfer learning for feature extraction are often showing higher performance than standard machine learning for handcrafted-feature classification [40]. This has a crucial role in the medical field, where achieving high classification performance is necessary but labeled datasets large enough to train a CNN model from scratch are challenging to collect $[3,40]$. In fact, the comparison between the results obtained when testing VGG 16 trained from scratch and VGG 16 pre-trained on ImageNet, showed that transfer learning helped in boosting performance.

\subsection{Impact of fine-tuning technique}

The fine-tuned VGG 16-based classification (C2 in Table 3) showed higher value of $\mathbf{R e c}$ for $\mathbf{I}$ and of $\mathbf{R e c}$ for $\mathbf{U}$ compared to SVMs classification, while the other two classes (i.e., B, S) achieved comparable results. One possible reason for this could be due to the relative small size of the dataset (less than a thousand samples), for which SVM may be more suitable [13], because of the particular ability at drawing decision boundaries on a small dataset.

Hence, as future work, we aim at enlarging the dataset exploiting Generative Adversarial Networks in order to enable better fine-tuning of the proposed system to potentially solve misclassification problems.

We are also interested in investigating contentious-learning strategies that use feedback from clinicians to train and tune the classification model in real time. Furthermore, we intend to explore the performance of the proposed algorithm when applied to endoscopy and microscopy videos of different anatomical regions, such as the gastro-intestinal tract.

\subsection{Experiment on a complete video sequence}

When testing the proposed algorithm on a fully labeled video sequence, misclassification of $\mathbf{I}$ frames occurred only with respect to $\mathbf{S}$ when there is a portion of leukoplakia in the image that, being white and homogeneous, is visually similar to specular reflections, and with respect to $\mathbf{U}$ frames. However, 
Table 7 Sample informative frames (I) and relative classification for each tested convolutional neural network (CNN). The red and green boxes correspond to misclassification and correct classification, respectively. $\mathbf{S}$ : frames with saliva or specular reflections, $\mathbf{U}$ : underexposed frames.

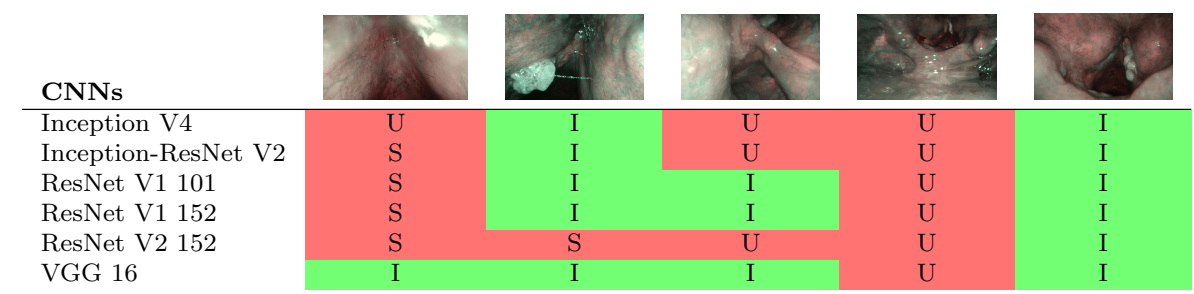

Table 8 Tested convolutional neural networks (CNNs) and corresponding number of informative frames (I) misclassified as frames with saliva or specular reflections (S) and as underexposed (U). No I frame was misclassified as blurred (B) by any CNN. The total number of informative frames is 180 .

\begin{tabular}{ccc}
\hline CNNs & $\begin{array}{c}\text { No. of I } \\
\text { misclassified as S }\end{array}$ & $\begin{array}{c}\text { No. of I } \\
\text { misclassified as U }\end{array}$ \\
\hline Inception V4 & 6 & 22 \\
Inception - ResNet V2 & 9 & 15 \\
ResNet V1 101 & 7 & 6 \\
ResNet V1 152 & 10 & 4 \\
ResNet V2 152 & 22 & 13 \\
VGG 16 & $\mathbf{0}$ & $\mathbf{5}$ \\
\hline
\end{tabular}

misclassification occurred primarily between classes of uninformative frames. These results demonstrated that in this video sequence, a total number of 146 uninformative frames out of 154 could be automatically removed.

\section{Conclusion}

This paper presented a method for endoscopic informative-frame classification that exploited CNN-based learned features through transfer learning and coupled with SVM multi-class classification, and classification after fine-tuning implementation on the pre-trained CNN. With our experimental protocol, the overall median classification recall among the four frame classes (i.e. B, I, $\mathbf{S}, \mathbf{U}$ ) for the best-performing learned features (VGG 16) set, coupled with transfer learning and SVM multi-class classification, was 93.61\% (max recall $=97.22 \%$ for the informative frames). The overall median recall among the four frame classes achieved with the fine-tuned VGG 16-based classification was $92.64 \%$ ( $\max$ recall $=97.78 \%$ for the informative frames). Both approaches outperformed the state of the art.

To conclude, this research demonstrated that using learned features obtained through transfer learning, together with SVMs or CNN-based classification, is an effective approach for the classification of informative frames in endoscopic videos. 


\section{References}

1. Maier-Hein L, Vedula SS, Speidel S, Navab N, Kikinis R, Park A, Eisenmann M, Feussner H, Forestier G, Giannarou S, et al (2017) Surgical data science for next-generation interventions. Nature Biomedical Engineering 1(9):691

2. Campochiaro PA (2015) Molecular pathogenesis of retinal and choroidal vascular diseases. Progress in Retinal and Eye Research

3. Moccia S, De Momi E, El Hadji S, Mattos LS (2018) Blood vessel segmentation algorithms - Review of methods, datasets and evaluation metrics. Computer Methods and Programs in Biomedicine 158:71-91

4. Piazza C, Del Bon F, Peretti G, Nicolai P (2012) Narrow band imaging in endoscopic evaluation of the larynx. Current Opinion in Otolaryngology \& Head and Neck Surgery 20(6):472-476

5. Moccia S, De Momi E, Guarnaschelli M, Savazzi M, Laborai A, Guastini L, Peretti G, Mattos LS (2017) Confident texture-based laryngeal tissue classification for early stage diagnosis support. Journal of Medical Imaging 4(3):034,502

6. Araújo T, Santos CP, De Momi E, Moccia S (2019) Learned and handcrafted features for early-stage laryngeal SCC diagnosis. Medical \& Biological Engineering \& Computing, 57(12):2683-2692.

7. Essert C, Fernandez-Vidal S, Capobianco A, Haegelen C, Karachi C, Bardinet E, Marchal M, Jannin P (2015) Statistical study of parameters for deep brain stimulation automatic preoperative planning of electrodes trajectories. International Journal of Computer Assisted Radiology and Surgery 10(12):1973-1983

8. Moccia S, Foti S, Routray A, Prudente F, Perin A, Sekula RF, Mattos LS, Balzer JR, Fellows-Mayle W, De Momi E, et al (2018) Toward improving safety in neurosurgery with an active handheld instrument. Annals of Biomedical Engineering pp 1-15

9. Gómez P, Semmler M, Sch'utzenberger A, Bohr C, D'ollinger M (2019) Low-light image enhancement of high-speed endoscopic videos using a convolutional neural network. Medical \& biological engineering \& computing pp $1-13$

10. Wang Z, Bovik AC, Sheikh HR, Simoncelli EP (2004) Image quality assessment: from error visibility to structural similarity. IEEE Transactions on Image Processing 13(4):600-612

11. Moccia S, Vanone GO, De Momi E, Laborai A, Guastini L, Peretti G, Mattos LS (2018) Learning-based classification of informative laryngoscopic frames. Computer Methods and Programs in Biomedicine 158:21-30

12. Perperidis A, Akram A, Altmann Y, McCool P, Westerfeld J, Wilson D, Dhaliwal K, McLaughlin S (2017) Automated detection of uninformative frames in pulmonary optical endomicroscopy. IEEE Transactions on Biomedical Engineering 64(1):87-98

13. Goodfellow I, Bengio Y, Courville A, Bengio Y (2016) Deep learning, vol 1. MIT press Cambridge 
14. Esteva A, Kuprel B, Novoa RA, Ko J, Swetter SM, Blau HM, Thrun S (2017) Dermatologist-level classification of skin cancer with deep neural networks. Nature 542(7639):115-118

15. Wang Q, Zheng Y, Yang G, Jin W, Chen X, Yin Y (2018) Multiscale rotation-invariant convolutional neural networks for lung texture classification. IEEE Journal of Biomedical and Health Informatics 22(1):184-195

16. Nanni L, Ghidoni S, Brahnam S (2017) Handcrafted vs. non-handcrafted features for computer vision classification. Pattern Recognition 71:158-172

17. Poplin R, Varadarajan AV, Blumer K, Liu Y, McConnell MV, Corrado GS, Peng L, Webster DR (2018) Prediction of cardiovascular risk factors from retinal fundus photographs via deep learning. Nature Biomedical Engineering $\mathrm{p} 1$

18. Russakovsky O, Deng J, Su H, Krause J, Satheesh S, Ma S, Huang Z, Karpathy A, Khosla A, Bernstein M, et al (2015) Imagenet large scale visual recognition challenge. International Journal of Computer Vision $115(3): 211-252$

19. Bashar MK, Kitasaka T, Suenaga Y, Mekada Y, Mori K (2010) Automatic detection of informative frames from wireless capsule endoscopy images. Medical Image Analysis 14(3):449-470

20. Atasoy S, Mateus D, Meining A, Yang GZ, Navab N (2012) Endoscopic video manifolds for targeted optical biopsy. IEEE Transactions on Medical Imaging 31(3):637-653

21. Park SY, Sargent D, Spofford I, Vosburgh KG, A-Rahim Y (2012) A colon video analysis framework for polyp detection. IEEE Transactions on Biomedical Engineering 59(5):1408

22. Maghsoudi OH, Talebpour A, Soltanian-Zadeh H, Alizadeh M, Soleimani HA (2014) Informative and uninformative regions detection in WCE frames. Journal of Advanced Computing 3(1):12-34

23. Ishijima A, Schwarz RA, Shin D, Mondrik S, Vigneswaran N, Gillenwater AM, Anandasabapathy S, Richards-Kortum R (2015) Automated frame selection process for high-resolution microendoscopy. Journal of Biomedi- cal Optics 20(4):046,014

24. Armin MA, Chetty G, Jurgen F, De Visser H, Dumas C, Fazlollahi A, Grimpen F, Salvado O (2015) Uninformative frame detection in colonoscopy through motion, edge and color features. In: International Workshop on Computer-Assisted and Robotic Endoscopy, Springer, pp 153-162

25. Kumar A, Kim J, Lyndon D, Fulham M, Feng D (2017) An ensemble of fine-tuned convolutional neural networks for medical image classification. IEEE Journal of Biomedical and Health Informatics 21(1):31-40

26. Yoo TK, Choi JY, Seo JG, Ramasubramanian B, Selvaperumal S, Kim DW (2019) The possibility of the combination of oct and fundus images for improving the diagnostic accuracy of deep learning for age-related macular degeneration: a preliminary experiment. Medical \& Biological Engineering \& Computing 57(3):677-687

27. Cheplygina V, Pena IP, Pedersen JH, Lynch DA, Sørensen L, de Bruijne M (2018) Transfer learning for multicenter classification of chronic obstruc- 
tive pulmonary disease. IEEE Journal of Biomedical and Health Informatics 22(5):1486-1496

28. Zhang R, Zheng Y, Mak TWC, Yu R, Wong SH, Lau JY, Poon CC (2017) Automatic detection and classification of colorectal polyps by transferring low-level CNN features from nonmedical domain. IEEE Journal of Biomedical and Health Informatics 21(1):41-47

29. Behrens A (2008) Creating panoramic images for bladder fluorescence endoscopy. Acta Polytechnica 48(3)

30. Yosinski J, Clune J, Bengio Y, Lipson H (2014) How transferable are features in deep neural networks? In: Advances in neural information processing systems, pp 3320-3328

31. Pan SJ, Yang Q (2009) A survey on transfer learning. IEEE Transactions on knowledge and data engineering 22(10):1345-1359

32. Krizhevsky A, Sutskever I, Hinton GE (2012) Imagenet classification with deep convolutional neural networks. In: Advances in Neural Information Processing Systems, pp 1097-1105

33. Simonyan K, Zisserman A (2014) Very deep convolutional networks for large-scale image recognition. arXiv preprint arXiv:14091556

34. Szegedy C, Ioffe S, Vanhoucke V, Alemi AA (2017) Inception-v4, inception-resnet and the impact of residual connections on learning. In: Association for the Advancement of Artificial Intelligence, vol 4, p 12

35. He K, Zhang X, Ren S, Sun J (2016) Deep residual learning for image recognition. In: IEEE Conference on Computer Vision and Pattern Recognition, pp 770-778

36. He K, Zhang X, Ren S, Sun J (2016) Identity mappings in deep residual networks. In: European Conference on Computer Vision, Springer, pp $630-645$

37. Burges CJ (1998) A tutorial on support vector machines for pattern recognition. Data Mining and Knowledge Discovery 2(2):121-167

38. Lin Y, Lv F, Zhu S, Yang M, Cour T, Yu K, Cao L, Huang T (2011) Large-scale image classification: fast feature extraction and SVM training. In: IEEE Conference on Computer Vision and Pattern Recognition, IEEE, pp 1689-1696

39. Tajbakhsh N, Shin JY, Gurudu SR, Hurst RT, Kendall CB, Gotway MB, Liang J (2016) Convolutional neural networks for medical image analysis: full training or fine tuning? IEEE transactions on medical imaging 35(5):1299-1312

40. Litjens G, Kooi T, Bejnordi BE, Setio AAA, Ciompi F, Ghafoorian M, Van der Laak JA, Van Ginneken B, Sánchez CI (2017) A survey on deep learning in medical image analysis. Medical Image Analysis 42:60-88

41. Esmaeili N, Illanes A, Boese A, Davaris N, Arens C, Friebe M (2019) Novel automated vessel pattern characterization of larynx contact endoscopic video images. International Journal of Computer Assisted Radiology and Surgery, pp $1-11$

42. Glorot X, Bengio Y (2010) Understanding the difficulty of training deep feedforward neural networks. In: Proceedings of the Thirteenth International 
Conference on Artificial Intelligence and Statistics, pp 249-256

43. Li X, La R, Wang Y, Niu J, Zeng S, Sun S, Zhu J (2019) EEG-based mild depression recognition using convolutional neural network. Medical \& Biological Engineering \& Computing 57(6):1341-1352

44. Dao TT (2019) From deep learning to transfer learning for the prediction of skeletal muscle forces. Medical \& Biological Engineering \& Computing 57(5):1049-1058

45. Singh R, Ahmed T, Singh R, Udmale SS, Singh SK (2019) Identifying tiny faces in thermal images using transfer learning. Journal of Ambient Intelligence and Humanized Computing, pp 1-10

46. Taylor ME, Stone P (2009) Transfer learning for reinforcement learning domains: A survey. Journal of Machine Learning Research 10(Jul):1633-1685

47. Pan SJ, Shen D, Yang Q, Kwok JT (2008) Transferring localization models across space. In: Proceedings of the Thirteenth International Conference on Artificial Intelligence, pp 1383-1388

48. Moccia S, Penza V, Vanone GO, De Momi E, Mattos LS (2016) Automatic workflow for narrow-band laryngeal video stitching. In: IEEE Annual International Conference of the Engineering in Medicine and Biology Society, pp $1188-1191$

49. Wilson AC, Roelofs R, Stern M, Srebro N, Recht B (2017) The marginal value of adaptive gradient methods in machine learning. In: Advances in Neural Information Processing Systems, pp 4148-4158

50. Bradley AP (1997) The use of the area under the ROC curve in the evaluation of machine learning algorithms. Pattern Recognition 30(7):1145-1159

Ilaria Patrini (B.Sc. 2016) is a M.Sc. student of Biomedical Engineering in the Electronic Information and Bioengineering Department (DEIB) of Politecnico di Milano. She is currently doing her M.Sc. thesis at the Neuroengineering and Medical Robotics Laboratory (NearLab).

Michela Ruperti (B.Sc. 2016) is a M.Sc. student of Biomedical Engineering in the Electronic Information and Bioengineering Department (DEIB) of Politecnico di Milano. She is currently doing her M.Sc. thesis at the Neuroengineering and Medical Robotics Laboratory (NearLab).

Sara Moccia (B.Sc. 2012, M.Sc. 2014, Ph.D. 2018) was born in Bari (Italy) on September 1990. She graduated cum laude in Biomedical Engineering at Politecnico di Milano (Milan, Italy) in December 2014, with a thesis entitled: "Statistical-segmentation techniques of liver metastases and necroses in FGDPET for the automatic evaluation of pre and post thermoablation PET/CT studies". In May 2018, she obtained the European PhD cum laude in Bioengineering from Istituto Italiano di Tecnologia, Department of Advanced Robotics (Genoa, Italy) and Politecnico di Milano, Department of Electronics, Information and Bioengineering with a thesis entitled "Supervised tissue classification in optical images: Towards new applications of surgical data science". During 
her $\mathrm{PhD}$, she was hosted at the Department of Computer-Assisted Medical Interventions at the German Cancer Research Center (Heidelberg, Germany). Sara is currently Postdoc at Università. Politecnica delle Marche, Department of Information Engineering (Ancona, Italy) and Affiliated Researcher at Istituto Italiano di Tecnologia.

Leonardo S. Mattos (B.Sc. 1998, M.Sc. 2003, Ph.D. 2007) is a Permanent Researcher and Head of the Biomedical Robotics Laboratory at the Italian Institute of Technology (IIT, Genoa). His research background ranges from robotic microsurgery and assistive human-machine interfaces to computer vision and micro-biomanipulation. Leonardo received his Ph.D. degree in electrical engineering from the North Carolina State University (NCSU, USA), where he worked as research assistant at the Center for Robotics and Intelligent Machines (CRIM) from 2002 until 2007. Since then he has been a researcher at the IITs Department of Advanced Robotics. Dr. Mattos leads a team of 22 researchers and technicians at IIT and collaborates closely with other institutions, including hospitals and industry. So far, Leonardo has been a key player in securing over 8 million Euros for research through externally funded projects. He was the PI and coordinator of the European project $\mu$ RALP Micro-Technologies and Systems for Robot-Assisted Laser Phonomicrosurgery, and is currently the PI and coordinator of the translational project Robotic Microsurgery and of the TEEP-SLA project, which is dedicated to the creation of new interfaces and assistive systems for ALS patients. Dr. Mattos is also participating to the project Sistemi Cibernetici Collaborativi - Teleoperation as coordinator and work package leader. He is the author or co-author of more than 130 peer-reviewed publications and has been the chair and main organizer of several international scientific events, including the 9th Joint Workshop on New Technologies for Computer/Robot Assisted Surgery (CRAS 2019), the 4th Joint Workshop on New Technologies for Computer/Robot Assisted Surgery (CRAS 2014), the IEEE BioRob 2014 Workshop on Robotic Microsurgery and Image-Guided Surgical Interventions, and the IEEE BioRob 2012 Workshop on Robot-Assisted Laryngeal Microsurgery. Leonardo has also been part of scientific and program committees of major international conferences, serving as Associate Editor for ICRA, BioRob, MFI, and the Hamlyn Symposium. He is also a regular reviewer for several computer-assisted surgery and robotics conferences and journals.

Emanuele Frontoni (M.Sc. 2003, Ph.D. 2006) is Associate Professor in the Department of Information Engineering of Università Politecnica delle Marche. He received the doctoral degree in electronic engineering from the University of Ancona, Italy, in 2003. In the same year he joined the Dept. of Ingegneria Informatica, Gestionale e dellAutomazione (DIIGA) at the Università Politecnica delle Marche, as a Ph.D. student in "Intelligent Artificial Systems". He obtained his PhD in 2006 discussing a thesis on Vision Based Robotics. His research focuses on applying computer science, artificial intelligence and com- 
puter vision techniques to mobile robots and innovative IT applications. He is a member of IEEE and $\mathrm{AI}^{*} \mathrm{IA}$, the Italian Association for Artificial Intelligence.

Elena De Momi (M.Sc. 2002, Ph.D. 2006) is Associate Professor in the Department of Electronic Information and Bioengineering (DEIB) of Politecnico di Milano. She is co-founder of the Neuroengineering and Medical Robotics Laboratory, in 2008, being responsible of the Medical Robotics section. IEEE Senior Member, she is currently Associate Editor of the Journal of Medical Robotics Research, of the International Journal of Advanced Robotic Systems, Frontiers in Robotics and AI and Medical \& Biological Engineering \& Computing. From 2016 she has been an Associated Editor of IEEE ICRA, IROS and BioRob, Area Chair of MICCAI and she is currently Publication Co-Chair of ICRA 2019. She is responsible for the lab course in Medical Robotics and of the course on Clinical Technology Assessment of the MSc degree in Biom. Eng. at Politecnico di Milano and she serves in the board committee of the $\mathrm{PhD}$ course in Bioengineering. Her academic interests include computer vision and image-processing, artificial intelligence, augmented reality and simulators, teleoperation, haptics, medical robotics, human robot interaction. She participated to several EU funded projects in the field of Surgical Robotics (ROBOCAST, ACTIVE and EuRoSurge, where she was PI for partner POLIMI). She is currently PI for POLIMI of the EDEN2020 project, aimed at developing a neurosurgery drug delivery system and of the ATLAS MSCA-ITN-2018-EJD, and coordinator of the MSCA-IF-2017 Individual Fellowships. She has been evaluator and reviewer for the European Commission in FP6, FP7 and H2020. 